Research Paper

\title{
Incidence, Clinical, Microbiological Features and Out- come of Bloodstream Infections in Patients Undergoing Hemodialysis
}

Maria Fysaraki², George Samonis ${ }^{1}$, Antonis Valachis' ${ }^{1}$ Eugenios Daphnis², Drosos E. Karageorgopoulos ${ }^{3,6,7}$, Matthew E. Falagas ${ }^{3,4,5}, K$ Kostas Stylianou², Diamantis P. Kofteridis ${ }^{\circledR}$

1. Department of Internal Medicine-Infectious Disease Unit University Hospital of Heraklion, Crete, Greece

2. Department of Nephrology, University Hospital of Heraklion, Crete, Greece

3. Alfa Institute of Biomedical Sciences (AIBS), Athens, Greece

4. Department of Medicine, Henry Dunant Hospital, Athens, Greece

5. Department of Medicine, Tufts University School of Medicine, Boston, Massachusetts, USA

6. Department of Medicine, Hygeia Hospital, Marousi, Athens, Greece

7. Hellenic Center for Disease Control and Prevention, Marousi, Athens, Greece

$\triangle$ Corresponding author: Diamantis P. Kofteridis, MD, Department of Internal Medicine, University Hospital of Heraklion, P.O. Box 1352, 71110 Heraklion, Crete, Greece. Tel: (+30) 2810 392688, Fax: (+30) 2810 392359, E-mail: kofterid@med.uoc.gr

(c) Ivyspring International Publisher. This is an open-access article distributed under the terms of the Creative Commons License (http://creativecommons.org/ licenses/by-nc-nd/3.0/). Reproduction is permitted for personal, noncommercial use, provided that the article is in whole, unmodified, and properly cited.

Received: 2013.05.16; Accepted: 2013.09.04; Published: 2013.09.20

\begin{abstract}
Objectives: Infection is a common cause of death among hemodialysis patients. The study investigated incidence, risk factors, clinical features and outcome of bloodstream infections (BSIs) in haemodialysis patients.

Methods: The records of haemodialysis patients from 1999 to 2005 were reviewed. Risk factors were investigated by multivariate analysis.

Results: There were identified I 48 bacteremic episodes, in 102 patients. The BSI rate was 0.52 per 1000 patient-days. Of the 148 episodes, 34 occurred in patients with permanent fistulae ( $0.18 / 1000$ patient-days); 19 in patients with grafts $(0.39 / 1000$ patient-days); 28 in patients with permanent tunneled central catheters (I.03/1000 patient-days); and 67 in those with temporary-catheter (3.18/1000 patient-days). With fistula as reference, the BSI ratio was 1.84 with arteriovenous graft $(P=.029), 4.85$ with permanent central venous catheter $(P<.00 I)$, and I 4.88 with temporary catheter $(P<.00 I)$. Catheter related were $4 \mathrm{I}$ episodes $(28 \%)$. Gram positive organism were responsible for 96 episodes $(65 \%)$, with S. aureus ( $55 \%$ ) the most frequent, followed by S. epidermidis (26\%) and Gram-negative for $36(23 \%)$, with $E$. coli (39\%) the most frequent. Infection was polymicrobial in $14(9.5 \%)$. Diabetes $(p<0.00 \mathrm{I})$, low serum albumin $(p=0.040)$ and low hemoglobin $(p<0.00 \mathrm{I})$ were significant risk factors. During hospitalization 18 patients $(18 \%)$ died. Septic shock $(p<0.00 \mathrm{I})$ and polymicrobial infection $(p=0.04 \mathrm{I})$ were associated with in-hospital mortality.
\end{abstract}

Conclusion: The risk of BSI in patients undergoing hemodialysis is related to the catheter type and vascular access. Septic shock and polymicrobial infection predispose to unfavourable outcome.

Key words: Antimicrobial Resistance, Sepsis, Vascular Catheter-Related Infections, Epidemiology

\section{Introduction}

Patients with end-stage renal disease (ERSD) requiring dialysis are at increased risk for bloodstream infection (BSI). This type of infection repre- sents a main cause of morbidity, as well as a preventable cause of death, along with increased costs and hospitalization [1-5]. 
Impaired immunity due to renal failure, comorbidities, malnourishment that increase the virulence and the adherence properties of hospital bacteria as well as the breakdown of the protective anatomical barriers due to repeated intravascular intervention required for haemodialysis, represent the main reasons for the high prevalence of bloodstream infection in those patients [3,6-8].

Previous studies suggested that the vascular access for hemodialysis is the major risk factor for bacteremia in patients with ESRD [9-12]. The infection risk has been found to be lower when the vascular access occurs through arteriovenous graft or fistula and higher when it occurs through central venous catheter (CVC), either permanent or temporary [9-12].

Chronic dialysis patients are at risk for infections caused by nosocomial multidrug resistant (MDR) pathogens exhibiting decreased susceptibility to many antimicrobials. Hence, empirical administration of such antimicrobials may be inappropriate resulting in increased morbidity, mortality and cost to the health care system [13]. Gram positive organisms are the most common etiologic agents of bacteremia in this setting; however, infections may also be caused by gram-negative pathogens [14-16]. The latter are of particular concern due to the absence of new drug development against them.

We, therefore, conducted a retrospective study in order to identify and compare the incidence of bacteremias associated with different types of vascular and non-vascular access and to determine risk factors, microbiological features and outcome of bacteremia among patients undergoing hemodialysis.

\section{Patients and Methods}

\section{Study Population}

The medical records of 239 patients undergoing hemodialysis at the Department of Nephrology of the University Hospital of Heraklion, Crete, Greece over a 7-year period (1999 to 2005) were retrospectively reviewed. Each patient was evaluated by both nurses and medical staff during each dialysis session for the presence of bloodstream infection (BSI) using CDC case definitions [17]. Only outpatients undergoing chronic hemodialysis were studied. Patients receiving short-term dialysis were excluded.

Hemodialysis patients with a documented BSI, defined as the isolation of a bacterial or fungal pathogen from at least one blood culture in the presence of clinical signs of infection, were selected as "cases". If potential skin contaminants (e.g., coagulase-negative Staphylococci (CNS), Corynebacteria, Propionibacterium spp., Bacillus spp., or Micrococci) were isolated, the episode was included in the study only when at least
2 blood culture sets yielded the same microorganism and the patient was showing clinical signs of infection. Since any given patient could have a BSI more than once, we used the term "episode of BSI" for each separate event.

All patients who underwent chronic hemodialysis during the study period and did not experience a bloodstream infection were considered "controls".

In the case-control study, only the first episode of bacteremia in a given patient was included in the analysis as case in order to preserve the independence of the values.

\section{Rates of bloodstream infections}

The patients were categorized into 1 of 4 current vascular access types: arterovenous fistula, arterovenous graft, temporary central venous catheter (CVC) or permanent tunneled central catheter. The total number of dialysis sessions was recorded on daily basis and grouped according to the 4 vascular access types.

For the calculation of the BSI incidence rates, the number of the BSI episodes during the study period were used as numerator and the total cumulative number of hemodialysis days for the same period (patient-days) as denominator. Rates were calculated separately for each type of vascular access. Rates of BSI were expressed as the number of infections per 1,000 patient-days.

\section{Data collection}

Clinical variables collected were age, gender, underlying diseases, cause of renal failure, comorbidities, length of time on renal replacement therapy, vascular access type, history of bacteremia, previous and current erythropoietin treatment, any iron supplementation, history of blood transfusions and dialysis procedures, risk factors for bacteremia (presence of central venous catheters, prior invasive procedures, surgical procedure within the past month, urinary tract catheter) and source of bacteremia.

White blood cell count, haemoglobin, serum C-reactive protein (CRP), erythrocyte sedimentation rate (ESR), serum ferritin, serum albumin, serum calcium and phosphate product and serum parathyroid hormone concentration were the laboratory values collected at the study entry.

Data on duration of hospital stay, empirical treatment (appropriate or inappropriate) and outcome (cure or death due to bacteremic episode) were also carefully collected.

\section{Definitions}

Catheter-related bacteremia was defined as the occurrence of a positive blood culture from the cath- 
eter with or without a positive peripheral blood culture, in the presence of systemic symptoms of infection with no other source of infection identified. Bacteremia was classified as primary in the absence of an identified source growing the same organism(s) as that recovered from blood. When the organism isolated from blood was the same as the organism causing an infection at another site, the BSI was classified as secondary. Exceptions to this were intravascular device-associated BSIs, all of which were classified as primary even if localized signs of infection were present at the access site [18].

Established definitions for sepsis and septic shock were followed [19].

Antimicrobial therapy was considered appropriate if the bacteria isolated were sensitive to at least one of the antimicrobials used as empirical therapy.

A bacteremic episode was considered polymicrobial if more than one organism was isolated in the same blood culture.

Established definitions for multidrug-resistant (MDR), extensively drug-resistant (XDR) and pandrug-resistant (PDR) bacteria were followed [20].

Regarding outcome, an episode was considered cured if resolution of the infection was noted, the patient had been discharged. Relapse was defined as recurrence of the infection due to the same organism occurring during the following four weeks after completion of antimicrobial therapy. A second (new) BSI episode was considered if a blood culture meeting the case definition criteria grew a different bacterial species or, in the case of same species, if sufficient time (more than 4 weeks) had elapsed without antimicrobial therapy, so that a relapse of the previous infection to be extremely unlikely.

Death was attributed to the infectious process if the patient died after a clinical course suggesting persistent infection and/or if the patient died during the phase of acute infection and death could not be clearly attributed to any other cause.

\section{Statistical analysis}

The incidence of infection rates, in terms of 1,000 patient-days, were calculated for each type of vascular access based on the collected data from the device-days monitoring forms and the confirmed BSIs.

A Poisson regression model was used to determine the incidence rate ratios and associated 95\% confidence intervals, with fistula used as the reference access category.

Categorical variables were evaluated using chi-square or Fischer's exact test for comparisons between groups. Continuous variables were compared using Student's t-test or Mann-Whitney U test for not normal distributions.
Multivariate analyses were performed using logistic regression model to test for: (i) an independent association with the development of bacteraemia; and (ii) an independent association with mortality. Variables found to be statistically significant $(P<.05)$ in the bivariate analysis were entered into the multivariable model.

Statistical analysis was performed using SPSS 16 (SPSS Inc, Chicago, IL); $P<.05$ was used to determine statistical significance.

\section{Results}

\section{Patient demographics}

During the 7-year study period, 148 bacteremic episodes, occurring in 102 patients, were identified. Their median age was 70 years (range 20-90). There were $53(52 \%)$ women. Seventy one out of 102 patients $(69 \%)$ had only one bacteraemic episode, 20 (20\%) two and $11(11 \%)$ three or four.

The aetiology of ESRD for the study population was diabetic nephropathy (20\%), glomerulonephritis $(13 \%)$, polycystic kidney disease $(9 \%)$, and/or unknown $(46 \%)$.

Table 1 shows characteristics of patients with BSIs.

Table I. Demographics of 102 patients with bloodstream infection

\begin{tabular}{ll}
\hline Characteristic & Number of patients (\%) \\
\hline Sex & $49(48)$ \\
Male & $53(52)$ \\
Female & $70[20-90]$ \\
Median age [range] & \\
Cause of ESRD & $20(19.6)$ \\
Diabetic nephropathy & $13(12.7)$ \\
Glomerulonephritis & $9(8.9)$ \\
Polycystic kidney disease & $8(7.8)$ \\
Obstructive nephropathies & $4(3.9)$ \\
Hypertensive nephropathy & $1(1)$ \\
Familial kidney disease & $47(46.1)$ \\
Unknown & \\
Underlying disease & $67(66)$ \\
Hypertension & $40(39)$ \\
Diabetes Mellitus & $21(21)$ \\
Heart Failure & $18(18)$ \\
Coronary Disease & $11(11)$ \\
Anemia & $11(11)$ \\
Chronic Obstructive Pulmonary Disease & $11(11)$ \\
Secondary Hyperparathyroidism & $11(11)$ \\
Cancer & $5(5)$ \\
Depression &
\end{tabular}




\section{Infection rates and comparisons between ac- cess types}

Regarding the type of vascular access, 107 patients $(37,5 \%)$ had AV fistulas, 32 (11\%) AV graft, 104 $(36 . \%)$ temporary and $42(15 \%)$ permanent catheter.

The 148 BSIs detected represent an incidence rate of 0.52 per 1,000 patient-days. Of the 148 episodes, 34 (23\%) involved permanent fistulae $(0.18 / 1,000$ patient-days); 19 (13\%) grafts $(0.39 / 1,000$ patient-days); $28(19 \%)$ permanent tunneled central catheter (1.03/1,000 patient-days); and 67 (45\%) temporary-catheter (3.18/1,000 patient-days).

With fistula as the reference vascular access type, results has shown that the incidence rate ratio for BSI was 1.84 with arteriovenous (AV) graft access $(P=.029), 4.85$ with permanent central venous catheter access $(P<.001)$, and 14.88 with temporary catheter access $(P<.001)$.

The incidence rate ratios associated with different types of vascular access are summarized in Table 2.

BSI was strongly associated with central venous catheter access. Only 24 (16\%) BSI episodes occurred in patients with an arteriovenous fistula or graft, compared with 124 (84\%) BSI episodes in patients with a catheter access $(P<.001)$.

The mean time to BSI from the beginning of the observation period were for AV fistula $48 \pm 33$ months, for AV graft $41 \pm 33$ months, for permanent catheter $28 \pm 32$ months and for temporary catheter $18 \pm 28$ days.

Table 2. Risk of bloodstream infection according to the type of vascular access

\begin{tabular}{llll}
\hline Type of access & $\begin{array}{l}\text { Number of } \\
\text { bloodstream } \\
\text { infections }\end{array}$ & IRR $(95 \%$ CI $)$ & P-value \\
\hline Temporary CVC & 67 & $14.88(10.18-22.20)$ & $<0.001$ \\
Permanent CVC & 28 & $4.85(3.0-7.85)$ & $<0.001$ \\
AV graft & 19 & $1.84(1.06-3.16)$ & 0.029 \\
AV fistula & 34 & 1 & \\
\hline
\end{tabular}

\section{Source of bacteremia and microbiology}

The BSI was considered to be primary in 76 (51\%) episodes, catheter-related in 41 (28\%), while 31 (21\%) episodes were secondary to other sources. The most frequent diagnoses were urinary tract infections (12 episodes; 39\%), respiratory tract infections (8; $26 \%)$, and skin/soft tissue infections (6; 19\%).

In 134 episodes $(90.5 \%)$ a single microorganism was isolated. In 96 of them $(65 \%)$ a single gram-positive organism was involved; more than half (55\%) of these episodes were caused by Staphylococcus aureus and $26 \%$ by Staphylococcus epidermidis. In 36 $(33 \%)$ episodes a single Gram-negative bacillus was involved. Escherichia coli accounted for more than one-third (39\%) of them followed by Klebsiella spp. (17\%), Acinetobacter spp. (14\%) and Enterobacter spp. (14\%). A gram-negative anaerobe (Bacteroides fragilis) was the cause of 1 episode, and Candida albicans of another one. Bacteremia was polymicrobial in 14 cases (9.5\%). The predominant strains isolated from polymicrobial events were Staphylococcus aureus (7 isolates; 50\%), Enterobacter spp. (3; 21\%), E. coli (3; $21 \%)$, Enterococcus faecalis $(3 ; 21 \%)$ and Pseudomonas aeruginosa $(3 ; 21 \%)$.

The organisms isolated are shown in Table 3.

Table 3. Organisms isolated from I 48 bacteremic episodes

\begin{tabular}{ll}
\hline Pathogens & No. of BSI episodes (\%) \\
\hline Gram-positive bacteria & $\mathbf{9 6}(\mathbf{6 5 )}$ \\
Staphylococcus aureus & $53(36)$ \\
Staphylococcus epidermidis & $25(17)$ \\
Enterococcus faecalis & $6(4)$ \\
Streptococcus spp. & $4(3)$ \\
Staphylococcus lugdunensis & $2(1)$ \\
Streptococcus agalactiae & $2(1)$ \\
Staphylococcus coag.-negative & $1(1)$ \\
Staphylococcus haemolyticus & $1(1)$ \\
Staphylococcus simuluns & $1(1)$ \\
Enterococcus faecium & $1(1)$ \\
Gram-negative bacteria & $\mathbf{3 6}(\mathbf{2 4})$ \\
Escherichia coli & $14(9)$ \\
Klebsiella spp. & $6(4)$ \\
Acinetobacter spp. & $5(3)$ \\
Enterobacter spp. & $5(3)$ \\
Pseudomonas aeruginosa & $3(2)$ \\
Stenotrophomonas maltophilia & $3(2)$ \\
Anaerobes & $\mathbf{1}(\mathbf{1 )}$ \\
Fungi & $\mathbf{1}(\mathbf{1 )}$ \\
Polymicrobial & $\mathbf{1 4}(\mathbf{9 )}$ \\
Staphylococcus aureus & $\mathbf{7}$ \\
Escherichia coli & $\mathbf{3}$ \\
Enterococcus faecalis & $\mathbf{3}$ \\
Enterobacter spp & $\mathbf{3}$ \\
Pseudomonas aeruginosa & $\mathbf{3}$ \\
Staphylococcus epidermidis & $\mathbf{3}$ \\
Staphylococcus haemoliticus & $\mathbf{2}$ \\
Klebsiella pneumonia & $\mathbf{1}$ \\
Corynebacterium spp & $\mathbf{1}$ \\
Streptococcus pneumonia & $\mathbf{1}$ \\
Proteus mirabilis & $\mathbf{1}$ \\
Candida albicans &
\end{tabular}


The total number of pathogens isolated, including both uni- and polymicrobial episodes was 162 . Forty out of 60 (67\%) Staphylococcus aureus isolates and 19 out of 28 (68\%) of Staphyloccocus epidermidis exhibited resistance to methicillin. Of the 48 gram-negative bacteria isolated $20(42 \%)$ exhibited resistance to piperacillin-tazobactam, $18(38 \%)$ to cefepime, $14(29 \%)$ to ceftazidime, amikacin and ciprofloxacin and $12(24 \%)$ to imipenem-cilastatin. Additionally, 3 out of 17 (18\%) Escherichia coli isolates produced extended spectrum $\beta$-lactamase. Finally, 3 out of 8 isolated (37.5\%) Enterobacter spp. strains, 4 out of $5(80 \%)$ Acinetobacter spp. strains, and 2 out of 7 (29\%) Klebsiella spp. strains were sensitive only to colistin.

\section{Risk factors for bacteremia}

There were 239 patients enrolled in the case-control study; 102 cases and 137 controls. Bivariate analysis of categorical variables identified that female gender [53 cases (52\%) vs. 52 controls (38\%)], presence of diabetes mellitus [40 (39\%) vs. $25(18 \%)]$ and absence of fistula [81 (79\%) vs. $64(47 \%)$ ] were more common among patients with BSI. Considering continuous variables, lower serum protein, albumin and serum haemoglobin levels, and higher white blood cell count were more frequently associated with BSI (Table 4).

Table 4. Bivariate analysis of risk factors for bloodstream infection in patients receiving hemodialysis

\begin{tabular}{|c|c|c|c|}
\hline Characteristic & $\begin{array}{l}\text { Patients without } \\
\text { bacteremia } \\
(\mathrm{N}=137)\end{array}$ & $\begin{array}{l}\text { Patients with } \\
\text { bacteremia } \\
(\mathrm{N}=102)\end{array}$ & P-value \\
\hline Age, years (SD) & $65.9(15.9)$ & $66.4(14.7)$ & 0.925 (NS) \\
\hline Male gender & $85(62)$ & $49(48)$ & 0.031 \\
\hline \multicolumn{4}{|l|}{ Comorbidity } \\
\hline Diabetes Mellitus & $25(18)$ & $40(39)$ & $<0.0001$ \\
\hline Malignancy & $6(4)$ & $11(11)$ & 0.057 (NS) \\
\hline \multicolumn{4}{|l|}{ Vascular access } \\
\hline A/V fistula & $73(53)$ & $21(20)$ & $<0.0001$ \\
\hline $\mathrm{A} / \mathrm{V}$ graft & $13(10)$ & $10(10)$ & \\
\hline Temporary catheter & $37(27)$ & $53(52)$ & \\
\hline Permanent catheter & $14(10)$ & $18(18)$ & \\
\hline $\begin{array}{l}\text { Current erythropoi- } \\
\text { etin treatment }\end{array}$ & $86(63)$ & $66(65)$ & 0.759 (NS) \\
\hline Current iron therapy & $106(77)$ & $71(70)$ & 0.176 (NS) \\
\hline $\begin{array}{l}\text { Surgical procedure } \\
\text { within } 1 \text { mo. }\end{array}$ & $9(7)$ & $9(9)$ & 0.500 (NS) \\
\hline Serum protein & $6.61(0.77)$ & $6.40(0.85)$ & 0.011 \\
\hline Serum albumin & $3.69(0.40)$ & $3.43(0.65)$ & $<0.0001$ \\
\hline WBC on admission & 7233 (3294) & $15780(6496)$ & $<0.0001$ \\
\hline Serum ferritin & $219,4(255.4)$ & $360.8(410.0)$ & $0.062(\mathrm{NS})$ \\
\hline Serum Ca & $9,04(1.19)$ & $8.96(1.10)$ & 0.482 (NS) \\
\hline Serum P & $4.72(1.63)$ & $4.82(1.69)$ & 0.472 (NS) \\
\hline $\mathrm{Hb}$ & $11.14(1.53)$ & $10.51(1.58)$ & 0.001 \\
\hline
\end{tabular}

In the multivariable logistic regression model, presence of diabetes mellitus (OR: 2.651; $\mathrm{P}<0.001$ ), absence of fistula (OR: 2.933; $\mathrm{P}=0.047$ ), low serum albumin (OR: 1.976; $\mathrm{P}=0.040)$ and low serum haemoglobin levels (OR: 1.354; $\mathrm{P}<0.001$ ) were all independently associated with occurrence of a BSI.

\section{Outcome and Predictors of Mortality}

One hundred thirty out of 148 episodes (88\%) have been cured, whereas death attributed to the BSI occurred in 18 episodes (12\%). Five episodes (3\%) relapsed.

The initial empirical antimicrobial therapy was appropriate in 116 episodes $(78 \%)$, resulting in an infectious mortality rate of $6 \%$ and inappropriate in 32 episodes $(22 \%)$ resulting in an infectious mortality rate of $34 \%(\mathrm{p}<0.001)$.

After multiple logistic regression, only 2 variables had been found to be independently associated with death in hemodialysis patients with BSI: the presence of septic shock on admission (OR: 174.446, 95\% CI: 24.360-1249.245, P<0.001) and a polymicrobial episode (OR: 8.934, 95\% CI: 1.039-73.029, $\mathrm{P}=0.041$ ).

\section{Discussion}

In the present retrospective study, diabetes mellitus, temporary CVC vascular access, low serum albumin and hemoglobin levels all were risk factors for occurrence of a BSI in patients with ESRD, while septic shock upon admission and polymicrobial BSI were associated with poor outcome. It is worth noting that $25 \%$ of the bacteremic episodes were caused by gram-negative pathogens and a substantial proportion of them were sensitive only to colistin.

Infection is an important cause of morbidity and mortality among patients with ESRD [1-5]. In the HEMO study an infectious etiology was responsible for $23 \%$ of all deaths among 1846 chronic hemodialysis patients, while the annual rate of the infection related hospitalization was 35\% [4]. Furthermore, data from the United States [Renal Data System (USRDS)] showed that infection was the leading cause of death among patients with ESRD, while the majority of deaths in these patients were due to bactere$\mathrm{mia} /$ sepsis [1].

Bacteremia is common in patients undergoing hemodialysis, and the CVC is the most common source of the infection $[4,6,7,17]$. In the HEMO study patients with bacteremia or sepsis represented $45 \%$ of those hospitalized due to infection, while $23 \%$ of them were considered having access related infection [4].

Since the number of patients that need renal replacement therapy continues to grow, the prevalence of BSIs is expected to rise as well. In the present study 
arteriovenous fistulas and grafts were associated with the lowest cumulative rates of infection, followed by permanent catheters, while temporary central venous catheters were associated with the highest ones. These results are consistent with findings reported in the literature [3,9-12]. It has been demonstrated recently that hemodialysis via catheter, compared with that via fistula or graft, increases not only the infection rates, but also mortality [21,22]. However, in the present series we could not reveal any significant difference in mortality between vascular access types probably due to the small number of deaths.

The majority of the present BSIs were caused by gram positive micro- organisms, while the two most common pathogens isolated were, not surprisingly, the skin-derived Staphylococcus aureus and Staphylococcus epidermis, a finding consistent with other investigators' experience $[8,9,14,15,22]$.

Considering other BSI risk factors, diabetes mellitus, low serum albumin and low haemoglobin levels have all been significantly associated with increased risk for bacteremia in the present series. All three have been identified as risk factors of bacteremia in a number of other studies [3,6,7]. It is well-known that diabetes mellitus leads to impaired immunologic defense [23] and in combination with the immunosuppression caused by uremia, may lead to increased risk for bacteremia [24]. In addition, in the present study low albumin level on admission has been shown also to be a risk factor for bacteremia. Low serum albumin may represent patient's malnutrition, a common problem in dialyzed populations, causing immune defect and facilitating bacteremia [25,26]. These data suggest that careful clinical evaluation, improved nutrition and diabetes management in hemodialysis patients may decrease the incidence of bacteremia.

The role of low haemoglobin level as risk factor is more difficult to be explained. However, a lower hemoglobin level may potentially be associated with risk of iron overload, which may lead to enhancement of bacterial growth and impairment of phagocytic function $[24,26]$. Previous studies have shown that iron overload, render hemodialysis patients more susceptible to bacteremia $[24,26]$. Furthermore, the potential role of haemoglobin in hemodialysis patients is supported by the study of Ma et al., which demonstrated the negative effect of low hematocrit on overall and infection-related survival in hemodialysis patients [27]. However, in the present study we did not find any difference between bacteremic and non-bacteremic patients regarding ferritin levels.

Infections in patients receiving hemodialysis are often caused by resistant pathogens, due to frequent hospital admissions and the also frequent need for antimicrobial therapy $[28,29]$. In the present study more than two thirds of the S. aureus and S. epidermidis isolates were methicillin-resistant. It is of note that the proportion of methicillin-resistant pathogens in our study is remarkably higher compared to other studies, suggesting that the use of vancomycin as first-line therapy may be necessary in our unit with regard to local patterns of resistance $[9,10,15]$. However, no vancomycin resistant staphylococci or enterococci have been isolated from the present series of patients.

One fourth of the bacteremic episodes were caused by gram-negative pathogens resistant to common antimicrobial agents, a proportion consistent with prior studies $[9,10,15,22]$. It is worth noting that a substantial percentage of the gram-negative isolates $(25 \%)$ were sensitive only to colistin.

Polymicrobial infections, represented $10 \%$ of the present bacteremic episodes.

The present mortality rates are consistent with those previously reported $[2,5,30]$. Septic shock on admission and a polymicrobial episode were predictors of poor outcome. Polymicrobial bacteremia has been suggested as predictor of adverse outcome in several studies [31,32], including immunocompromised patients [33] but this is the first study which outlines its predictive value in hemodialysis patients and this should be taken into account when empirical antimicrobial therapy is undertaken.

The present study has some limitations: its retrospective nature makes it vulnerable to collection bias and to potential inaccuracy in data collection. Additionally the number of deaths was relatively small, leading to wide confidence intervals.

In conclusion, the risk of bacteremia in hemodialysis patients can be stratified according to vascular access type. Diabetes mellitus, low albumin and haemoglobin levels upon admission are independent risk factors for BSI, while the presence of septic shock and polymicrobial bacteremia predispose to unfavourable outcome. Hence, careful clinical evaluation, limitation of the catheter use, improvement of nutritional status, correction of anemia and successful management of diabetes mellitus considerably reduce the incidence of BSIs. Finally, surveillance of local microbiology is of utmost importance for appropriate empirical antimicrobial treatment.

\section{Competing Interests}

The authors have declared that no competing interest exists.

\section{References}

1. [Internet] US Renal Data System (The National Institutes of Health, National Institute of Diabetes, Digestive and Kidney Diseases, Bethesda, MD). USRDS 2008 Annual Data Report. http://www.usrds.org/2008/pdf/V2_06_2008.pdf. 
2. Tozawa M, Iseki K, Fukiyama K. Prevalence of hospitalization and prognosis of patients on chronic dialysis. Clin Exp Nephrol 2000;4:236-240

3. Hoen B, Paul-Dauphin A, Hestin D, et al. A multicenter prospective study of risk factors for bacteremia in chronic hemodialysis patients. J Am Soc Nephrol 1998;9:869-876

4. Allon M, Depner TA, Radeva M et al. Impact of dialysis dose and membrane on infection-related hospitalization and death: results of the HEMO Study. J Am Soc Nephrol 2003;14:1863-1870

5. Liu JW, Su YK, Liu CP, et al. Nosocomial blood-stream infections in patients with end-stage renal disease; excess length of hospital stay, extra cost and attributed mortality. Hosp Infect 2002;50:224-227

6. Powe NR, Jaar B, Furth SL. Septicemia in dialysis patients: incidence, risk factors, and prognosis. Kidney Int 1999;55:1081-1090

7. Jean G, Charra B, Chazot C, et al. Risk factor analysis for long-term tunnelled dialysis catheter-related bacteremias. Nephron 2002;91:399-405

8. Jaber BL. Bacterial infections in hemodialysis patients: pathogenesis and prevention. Kidney Int 2005;67:2508-2519

9. Taylor G, Gravel D, Johnston L, et al. Prospective surveillance for primary bloodstream infections occurring in Canadian hemodialysis units. Infect Control Hosp Epidemiol 2002;23:716-720

10. Dopirak M, Hill C, Oleksiw M, et al. Surveillance of hemodialysis-associated primary bloodstream infections: the experience of ten hospital based centers. Infect Control Hosp Epidemiol 2002;23:721-724

11. Tokars JI, Miller ER, Stein G. A new national surveillance system for hemodialysis-associated infections: initial results. Am J Infect Control 2002;30:288-295

12. Stevenson KB, Adcox MJ, Mallea MC, et al. Standardized surveillance of hemodialysis vascular access infections: 18-month experience at an outpatient, multifacility hemodialysis center. Infect Control Hosp Epidemiol 2000;21:200-203

13. Kollef $\mathrm{MH}$. Inadequate antimicrobial treatment: an important determinant of outcome for hospitalized patients. Clin Infect Dis 2000;31(Suppl 4):S131-138

14. Blakestijn PJ. Treatment and prevention of catheter-related infections in haemodialysis patients. Nephrol Dial Transplant 2001;16:1975-1978

15. Colville LA, Lee AH. Retrospective analysis of catheter-related infections in a hemodialysis Unit. Infect Control Hosp Epidemiol 2006;27:969-973

16. Marr KA, Kong LK, Fowler VG, et al. Incidence and outcome of Staphylococcus aureus bacteremia in hemodialysis patients. Kidney Int 1998;54:1684-1689

17. NKF-K/DOQI. Clinical practice guidelines for vascular access: update 2006. III. Prevention of complications: infection. National kidney foundation. Am J Kidney Dis 2006;48 (Suppl 1):S248-S273

18. [Internet] Centers of Disease Control and Prevention (CDC). Central-Line Associated Bloodstream Infection (CLA-BSI) event 2009. www.cdc.gov/nhsn/PDFs/pscManual/4PSC CLABScurrent.pdf

19. American College of Chest Physicians/ Society of Critical Care Medicine consensus conference: definitions for sepsis and organ failure and guidelines for the use of innovative therapies in sepsis. Crit Care Med 1992;20:864-874

20. Magiorakos AP, Srinivasan A, Carey RB, et al. Multidrug-resistant, extensively drug-resistant and pandrug-resistant bacteria: an international expert proposal for interim standard definitions for acquired resistance. Clin Microbiol Infect. 2012;18:268-81.

21. Thomson PC, Stirling CM, Geddes CC, et al. Vascular access in haemodialysis patients: a modifiable risk factor for bacteraemia and death. Q J Med 2007;100:415-422

22. Dhingra RK, Young EW, Hulbert-Shearson TE, et al. Type of vascular access and mortality in U.S. hemodialysis patients. Kidney Int 2001;60:1443-1451

23. Geerlings E, et al. Immune dysfunction in patients with diabetes mellitus (DM). FEMS Immunology and Medical Microbiology 1999;26: 259-265

24. Waterlot $Y$, Cantinieaux B, Hariga-Muller C, et al. Impaired phagocytic activity of neutrophils in patients receiving haemodialysis: the critical role of iron overload. Br Med J (Clin Res Ed). 1985;291(6494):501-504

25. Cianciaruso B, Brunori G, Traverso G, et al: Nutritional status in the elderly patient with uraemia. Nephrol Dial Transplant 1995;10 (suppl 6):65-68

26. Boelaert JR, Daneels RF, Schurgers ML, et al. Iron overload in haemodialysis patients increases the risk of bacteraemia: A prospective study. Nephrol Dial Transplant 1990;5:130-134

27. Ma JZ, Ebben J, Xia H, et al. Hematocrit level and associated mortality in hemodialysis patients. J Am Soc Nephrol. 1999;10:610-619

28. Montecalvo MA, Shay DK, Patel P, et al. Bloodstream infections with vancomycin-resistant enterococci. Arch Intern Med 1996;156:1458-1462
29. Smith TL, Pearson ML, Wilcox KR, et al. Emergence of vancomycin resistance in Staphylococcus aureus: Glycopeptide-Intermediate Staphylococcus aureus Working Group. N Engl J Med 1999;340:493-501

30. Saxena AK, Panhotra BR. Haemodialysis catheter-related bloodstream infections: current treatment options and strategies for prevention. Swiss Med Wkly. 2005;135:127-138

31. Pittet D, Li N, Wenzel RP. Association of secondary and polymicrobial nosocomial bloodstream infections with higher mortality. Eur J Clin Microbiol Infect Dis. 1993;12:813-819

32. Lin JN, Tsai YS, Lai CH, et al. Risk factors for mortality of bacteremic patients in the emergency department. Acad Emerg Med. 2009;16:749-755

33. Nørgaard M, Larsson H, Pedersen G, et al. Risk of bacteraemia and mortality in patients with haematological malignancies. Clin Microbiol Infect. 2006;12:217-223 